\title{
Abordagem cirúrgica para mucocele de tamanho atípico: relato de caso
}

Surgical approach for atypical size mucocelles: clinical case

Enfoque quirúrgico para mucocele de tamaño atípico: reporte de caso

Lucas Emmanuell de Morais NEVES ${ }^{1}$
Thálison Ramon de Moura BATISTA
Ruth Venâncio Fernandes DANTAS ${ }^{2}$
Clenia Emanuela de Sousa ANDRADE ${ }^{3}$
Ana Karoline Vieira MELO

${ }^{1}$ Hospital da Restauração - UPE/FOP, Residente em Cirurgia e Traumatologia Buco-maxilo-facial - Hospital da Restauração - UPE/FOP ${ }^{2}$ Graduando(a) do curso de Odontologia, Departamento de Odontologia, Universidade Estadual da Paraíba - UEPB / Campus VIII, 58233-000 Araruna - PB, Brasil ${ }^{3}$ Cirurgiã-Dentista pela Universidade Estadual da Paraíba-UEPB/ Campus VIII, 58233-000 Araruna-PB, Brasil

${ }^{4}$ Mestranda em Ciências Odontológicas pela Universidade Federal da Paraíba - 58051-900 João Pessoa - PB, Brasil

\section{Resumo}

Introdução: A Mucocele Oral (MO) é uma lesão pseudocística de retenção não neoplásico, comum das glândulas salivares menores, apresentando diversos fatores etiologicos, sendo o trauma e a inflamação do ducto salivar os mais frequentes. Objetivo: Reportar um caso de remoção cirúrgica de mucocele de tamanho atípico. Relato de caso: Paciente 27 anos, masculino, compareceu a uma clínica escola odontológica, relatando presença de lesão indolor em mucosa labial inferior, persistindo há aproximadamente duas semanas, com história previa de trauma por mordedura acidental. Ao exame físico observou-se lesão nodular de base séssil, normocorada, flácida à palpação, medindo aproximadamente $03 \mathrm{~cm}$ em seu maior diâmetro, em mucosa labial inferior. Estabeleceu-se a hipótese diagnóstica de mucocele, optando-se, pela remoção cirúrgica sob anestesia local, do bloqueio do nervo mentoniano unilateral. Realizou-se uma incisão linear em epitélio, seguida de meticulosa divulsão, tornando possível exérese de lesão, mantendo-se a integridade da cápsula. Por fim, realizou-se a sutura contínua simples da mucosa. Paciente continua em acompanhamento pós-operatório, sem sinal de recidiva. Conclusão: No caso clínico aqui relatado a remoção completa da mucocele tamanho atípico e glândulas salivares acessórias, bem como a ausência de recidivas, caracterizou o sucesso na abordagem do caso.

Descritores: Mucocele; Glândulas Salivares; Cirurgia; Incisão Cirúrgica.

\section{Abstract}

Introduction: Oral Mucocele (MO) is a non-neoplastic retention pseudocystic lesion, common in the minor salivary glands, presenting several etiologic factors, with trauma and inflammation of the salivary duct being the most frequent. Objective: To report a case of surgical removal of an atypical size mucocele. Case report: A 27-year-old male patient attended a dental school clinic, reporting the presence of a painless lesion in the lower lip mucosa, persisting for approximately two weeks, with a previous history of accidental bite trauma. On physical examination, a nodular lesion with a sessile base, normal color, flaccid on palpation was observed, measuring approximately $03 \mathrm{~cm}$ in its largest diameter, in the lower lip mucosa. The diagnostic hypothesis of mucocele was established, opting, by surgical removal under local anesthesia, for unilateral mental nerve block. A linear epithelial incision was performed, followed by meticulous divulsion, making possible excision of the lesion, maintaining the integrity of the capsule. Finally, simple continuous suture of the mucosa was performed. Patient continues to be monitored postoperatively, with no sign of recurrence. Conclusion: In the clinical case reported here, the complete removal of the atypical size mucocele and accessory salivary glands, as well as the absence of recurrences, characterized the success in approaching the case.

Descriptors: Mucocele; Salivary Glands; Surgery; Surgical Incision.

\section{Resumen}

Introducción: El mucocele oral (MO) es una lesión pseudoquística de retención no neoplásica, común en las glándulas salivales menores, que presenta varios factores etiológicos, siendo el trauma y la inflamación del conducto salival los más frecuentes. Objetivo: reportar un caso de extirpación quirúrgica de un mucocele de tamaño atípico. Informe del caso: un paciente masculino de 27 años asistió a una clínica de la escuela de odontología, informando la presencia de una lesión indolora en la mucosa del labio inferior, que persiste durante aproximadamente dos semanas, con antecedentes de trauma accidental por mordida. En el examen físico, se observó una lesión nodular con una base sésil, color normal, flácida a la palpación, que mide aproximadamente $03 \mathrm{~cm}$ en su diámetro más grande, en la mucosa del labio inferior. La hipótesis diagnóstica del mucocele se estableció, al optar, por la extirpación quirúrgica bajo anestesia local, por el bloqueo unilateral del nervio mental. Se realizó una incisión epitelial lineal, seguida de una divulgación meticulosa, haciendo posible la escisión de la lesión, manteniendo la integridad de la cápsula. Finalmente, se realizó una sutura continua simple de la mucosa. El paciente continúa siendo monitoreado después de la operación, sin signos de recurrencia. Conclusión: en el caso clínico reportado aquí, la extirpación completa del mucocele de tamaño atípico y las glándulas salivales accesorias, así como la ausencia de recurrencias, caracterizaron el éxito al abordar el caso.

Descriptores: Mucocele; Glándulas Salivales; Cirugía; Incisión Quirúrgica.

\section{INTRODUÇÃO}

A Mucocele Oral (MO) é uma lesão pseudocísticas de retenção não neoplásico, comum das glândulas salivares menores, apresenta diversos fatores etiologicos, sendo o trauma e a inflamação do ducto salivar os mais frequentes, acometendo em sua maior apresentações a mucosa interna do lábio inferior, seguido de assoalho da boca e ventre de lingua ${ }^{1-4}$. MO pode se desenvolver em qualquer idade e sem predileção por sexo ${ }^{3,4}$. Clinicamente é caracterizada como uma lesão exofítica, de aspecto nodular liso, esférico, assintomatico, de coloração que varia de azulado para translúcida e variando de $0,3 \mathrm{~cm}$ a $01 \mathrm{~cm}^{2,3}$.
As abordagens terapeuticas para MO incluem excisão cirúrgica, laserterapia, crioterapia, injeção de esteróides e micro-marsupialização, apresentando normalmento o prognóstico favoravel após exerese de lesão, com possibilidade de recorrência ${ }^{2,1}$.

Ante o exposto, o propósito do presente artigo é descrever a conduta de exérese cirúrgica de mucocele oral de tamanho atípico localizado em mucosa labial inferior.

\section{CASO CLÍNICO}

Paciente do sexo masculino, 27 anos, pardo, buscou um serviço de aperfeiçoamento de cirurgia 
oral menor, em João Pessoa - PB, motivada por uma lesão de rápido crescimento, em região de mucosa labial inferior, ocasionando leve desconforto e sintomatologia dolorosa ao trauma local, tal como mastigação. Clinicamente, observou-se lesão única, assintomática ao toque, com aproximadamente três centímetros em seu maior diâmetro, de base séssil, normocorada, de consistência firme e superfície lisa (Figura 1).

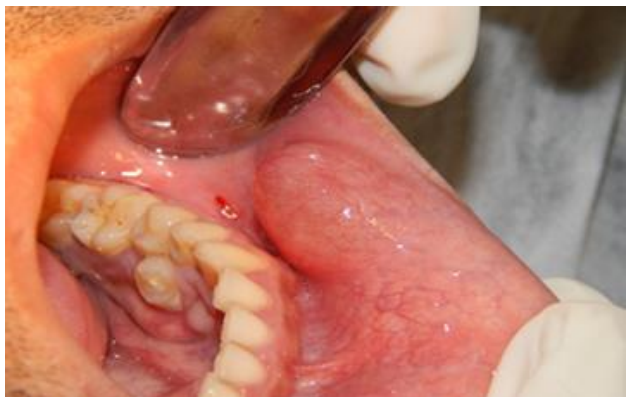

Figura 1: Aspecto clínico inicial da MO em mucosa labial inferior.

As hipóteses diagnósticas foram de mucocele oral e lipoma. Por tais hipóteses, optou-se por realizada uma biópsia excisional com finalidade diagnóstica e terapêutica. O procedimento cirúrgico foi realizado sob anestesia local por meio do bloqueio do nervo mentoniano unilateral com anestésico do tipo Articaína a $4 \%$ com adrenalina (Articaine), Seguiu-se a incisão retilínea em mucosa (Figura 2A) utilizando lâmina de bisturi de aço inox estéril $\mathrm{N}^{\circ} 15$

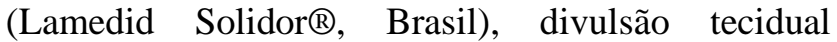
minuciosa (Figura 2B, 2C e 2D) e exérese da lesão(Figura 3A).

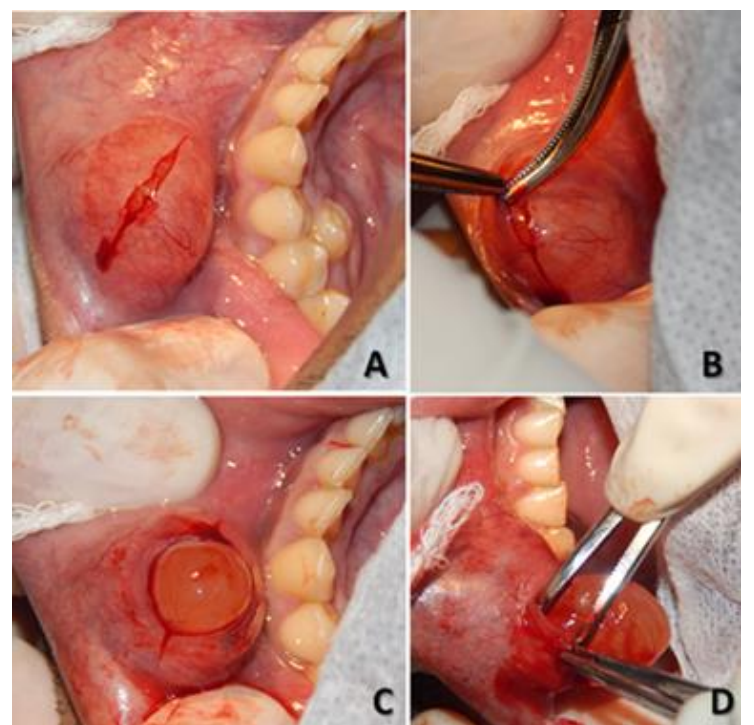

Figura 2. A:Incisão com lâmina de bisturi $\mathrm{N}^{\circ} 15$. B: Divulsão tecidual inicial para exposição de lesão. C: MO exposta através da incisão cirúrgica. D: Divulsão tecidual cuidadosa para não romper a cápsula da lesão.

Em sequência, foi realizado a limpeza copiosa com soro fisiológico 0,9\% (Figura 3 B) e síntese tecidual por primeira intenção com suturas simples sob fio de sutura Nylo 3.0mm (Figura 4). Por fim, a peça foi enviada para análise histopatológica (Figura 3C), obtendo como diagnóstico mucocele por extravasamento de muco. O paciente continua em acompanhamento sem sinal de recidiva da lesão e prognostico satisfatório.

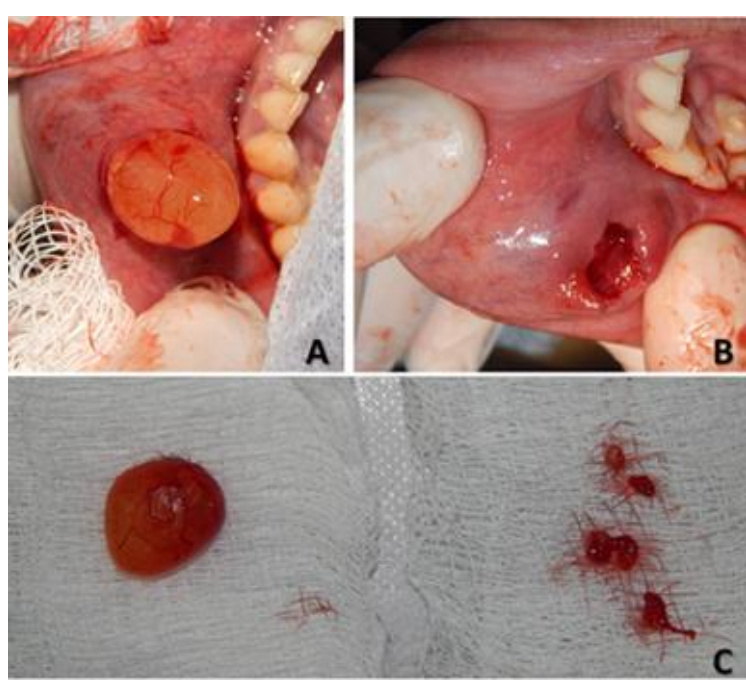

Figura 3. A: Exérese total de lesão com cápsula de revestimento integra. B. Aspecto de loja cirúrgica após revestimento de mucocele oral e glândulas salivares menores e lavagem de sítio cirúrgico com soro fisiológico 0,9\%. C. Lesão enviada para análise histopatológica.

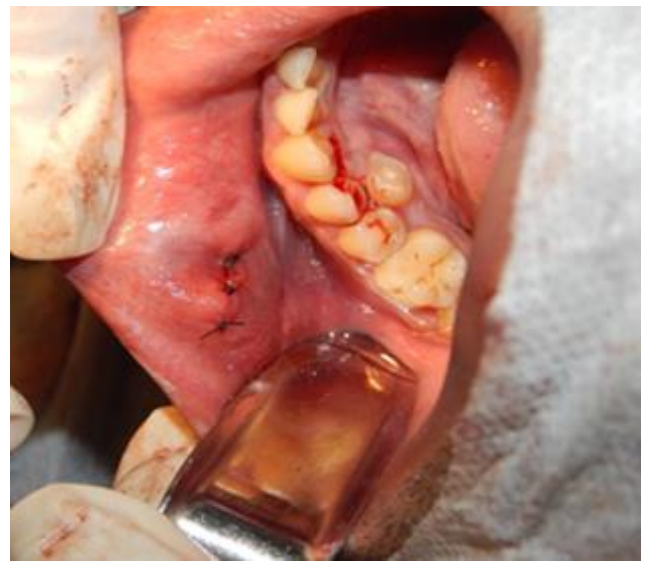

Figura 4. Aspecto pós-operatorio imediato. Sutura em ponto simples sem sinal de deiscência ou debito.

\section{DISCUSSÃO}

Mucocele é um termo híbrido, pois deriva do latin muco (muco) e do grego kele (acúmulo, hérnia, tumor), que descreve um fenômeno de extravasamento ou de retenção de muco ${ }^{5,6}$.

Assim como no caso aqui relatado, a literatura descreve maior acometimento em pacientes jovens. Segundo Choi et al. ${ }^{2}$ em seu estudo cujo objetivo foi identificar as possíveis variáveis preditivas que afetam a taxa de recorrência da mucocele oral, a maioria das lesões avaliadas foram observadas em pacientes com idade variando de 20 a 29 anos. Essas distribuições de idade podem estar relacionada à maior prevalência de hábitos parafuncionais durante essa faixa etária.

A incidência da mucocele oral entre os sexos permanecem inconclusivas ${ }^{7,8}$. Zanotto et al. ${ }^{9}$ em um levantamento das lesões provenientes das glândulas salivares menores diagnosticadas descreve uma predileção pelo gênero masculino $(50,84 \%)$, sem no 
entanto significância estatística. Em contrapartida, estudo de Bezerra et al. ${ }^{1}$ evidenciou maior frequência da lesão no sexo feminino $(54,7 \%)$.

Assim como visto neste relato de caso, vários estudos epidemiológicos publicados embasam que o lábio inferior é o sítio anatômico da cavidade oral mais acometido seguido da mucosa ventral da língua, mucosa jugal e parte superior mucosa labial ${ }^{5,2,9,10}$.

$\mathrm{O}$ diagnóstico de mucocele pode obtido por meio das suas características clínicas baseado na história da lesão, no entanto, pode haver casos em que sejam confundidas com outras patologias presentes na cavidade oral, sendo então, importante o envio da peça para análise histopatológica, a fim de confirmar a suspeita clínica ${ }^{1,6,7}$.

Assim como realizado no relato do caso aqui descrito, o estudo de Zanotto et al. ${ }^{9}$ (2019) descreve uma alta taxa de concordância observada entre o diagnóstico clínico e o histológico. Esses resultados corroboram com a atual literatura, onde sugere que o diagnóstico clínico para tal lesão, em sua maior frequência, não oferece grandes dificuldades.

Os aspectos clínicos da mucocele oral podem variar de acordo com a profundidade da lesão ${ }^{1,7,9}$. Lesões superficiais possuem característica clínica de vesícula ou bolha com tumefação arredondada, assintomática e superfície lisa ${ }^{1,7}$.

Em contrapartida, as mais profundas dão origem às mucoceles clássicas, apresentando-se como um nódulo bem circunscrito, recoberto por mucosa oral normal. Normalmente, não apresentam alterações de coloração da mucosa, possuem maiores dimensões e são mais firmes à palpação ${ }^{1,7}$.

As características clínicas do caso aqui descrito entram em concordância com a atual literatura. Bezerra et al. ${ }^{1}$ descrevem os aspecto clínico mais frequente da mucocele oral como uma lesão nodular (59,8\%), assintomática (70,9\%), normocorada $(36,2 \%)$; de superfície lisa $(13,1 \%)$ e medindo em sua maior extensão $0,9 \mathrm{~cm}$.

O tamanho da lesão aqui apresentado nesse relato de caso, chama atenção pelo fato de que normalmente as mucoceles são descritas na literatura variando de alguns milímetros a pouco mais de um centímetro, dependendo do tempo de evolução da lesão ${ }^{2,9}$.

No estudo de Oliveira et al. ${ }^{10}$, cerca de $75 \%$ dos casos de mucocele possuíram menos de $1 \mathrm{~cm}$ de diâmetro. Bezerra at al. ${ }^{1}$ apontam a faixa entre 0 até no máximo $2 \mathrm{~cm}$ como a mais comum $(86,50 \%)$.

Existem várias opções de tratamento para mucocele oral, incluindo excisão cirúrgica, marsupialização, criocirurgia e injeção de esteroides ${ }^{2}$. No entanto, o tratamento mais descrito na literatura é constituído pela excisão cirúrgica total da lesão juntamente com as glândulas salivares menores ${ }^{7,9}$.

O tratamento conservador é relatado para as lesões de discreta extensão no seu maior diametro, bem como as superficiais. Tal fato se justifica devido a resolubilidade espontânea da lesão ou ruptura da mesma por trauma local ${ }^{1,6,9,10}$.

Apesar do prognóstico dessa patologia ser excelente, são relatados na literatura casos de recorrência após abordagem terapeutica ou resolução espontânea ${ }^{7}$. No estudo de Choi et al. ${ }^{2}$ os autores descrevem uma recorrência de $12,8 \%$, sendo mais comum no primeiro mês após a remoção cirúrgica $(57,1 \%)$.

Qualquer glândula salivar secundária periférica deve ser removida para reduzir o risco de recorrência. As suturas também devem ser colocadas com cuidado para evitar lesões em outra glândula adjacente $^{6}$.

\section{CONCLUSÃO}

A mucocele oral é uma lesão benigna, sem preferencia por sexo e com maior frequência em pacientes jovens, com predileção da mucosa labial inferior, sofrendo influência direta de trauma local. O diagnóstico é facilmente obitido por meio de exame clínico, apresentando-se como uma lesão exofítica, nodular, assintomática, superfície lisa, normocorado e de pequeno diâmetro. A exérese da glândula salivar ainda é a abordagem terapêutica mais empregada. No caso clínico aqui relatado a remoção completa da mucocele e glândulas salivares acessórias, bem como a ausência de recidivas, caracterizou o sucesso na abordagem do caso.

\section{REFERÊNCIAS}

1. Bezerra TMM, Monteiro BVB, Henriques ACG, Carvalho MV, Nonaka CFW, Miguel MCC. Epidemiological survey of mucus extravasation phenomenon at an oral pathology referral center during a 43 year period. Braz J Otorhinolaryngol. 2016; 82(5):536-42.

2. Choi YJ, Byun JS, Choi JK, Jung JK. Identification of predictive variables for the recurrence of oral mucocele. Med oral patol oral cir bucal. 2019;24(2):e231-35.

3. Liu JL, Zhang AQ, Jiang LC, Li KY, Liu FZ, Yuan DY et al. The efficacy of polidocanol sclerotherapy in mucocele of the minor salivary gland. J Oral Pathol Med. 2018;47(9):895-99.

4. Titsinides S, Kalyvas D, Tosios K. Mucocele of the dorsal surface of the tongue: A case report. J Clin Exp Dent. 2018;10(5):e495-8.

5. Abdel-Aziz M, Khalifa B, Nassar A, Kamel A, Naguib N, El-Tahan AR. Mucocele of the hard palate in children. Int J Pediatr Otorhinolaryngol. 2016;85:46-49.

6. Lucero RAC, Araceli GR, Cesar BFJ. Fenómeno de extravasación mucosa. Rev Tamé. 2019; 7(21):835-37.

7. Balan I, Camargo WR, Ribas MB, Navarro $\mathrm{CH}$, Lobo F. Tratamento de mucocele com a técnica de Shira: relato de caso. Rev Odontol Araçatuba. 2019;40(2):54-8. 
8. Prosdócimo ML, Agostini M, Romañach MJ, de Andrade BAB. A retros-pective analysis of oral and maxillofacial pathology in a pediatric population from Rio de Janeiro-Brazil over a 75-year period. Med Oral Patol Oral Cir Bucal. 2018; 23(5):e511-7.

9. Zanotto PG. Levantamento dos casos de mucocele e rânula diagnosticados pela laboratório de patologia bucal da Universidade Federal de Santa Catarina entre 2006 e 2018 [monografia]. Florianópolis: Universidade Federal de Santa Catarina - UFSC; 2019.

10. Oliveira BF, Henrique DBB, Cruz JHA. Mucocele oral provocada por mordida acidental: relato de caso. Arch Health Investigation. 2019;7(11):455-60.

\section{CONFLITO DE INTERESSES}

Os autores declaram não haver conflitos de interesse.

\section{AUTOR PARA CORRESPONDÊNCIA}

\section{Thálison Ramon de Moura Batista}

thalison.rr@hotmail.com
Submetido em 02/04/2020

Aceito em 21/07/2020 